\title{
Medical Values from the Perspective of Cultural Tradition and Policy Intervention: Based on Chinese Theories and Empirical Research
}

\author{
Huan Liu* \\ School of Public Administration, Zhejiang University of Finance \& Economics, Hang Zhou, \\ China \\ Email: zcliuhuan@126.com \\ *Corresponding Author
}

\begin{abstract}
Background With the development of the medical security system, the problem of "difficult and expensive to see a doctor" has been greatly improved for Chinese residents. But at the same time, the Chinese people's view of value medical care has also taken on a great change, and then transferred to medical treatment behaviors and medical decision-making. In order to reveal the evolution mechanism of medical behavior and decision-making, this paper combines the concept of "taboo disease and taboo medical treatment" under traditional culture environment with the evolution of the medical concept under the protection of policies, and explores the impact of the two on transforming the concept of value medical treatment, establishing more scientific disease diagnosis and health concept.

Methods Based on the data of the Chinese Health Service Survey and the CLHLS survey database, this paper empirically tests the impact of traditional culture environment and policy
\end{abstract}


protection on value medical care from the perspective of disease occurrence and the laws of life course.

Results The results show that cultural tradition environments have a greater impact on the medical values of urban residents than that of rural residents, and the income level has significantly increased the level of medical expenditures one month before the end of life. After the instrumental variables are processed, the income level has no significant effect on the death probability of the elderly in medical institutions. Borne by the spouse or children living together or subsidized by the state or collective, the elderly who have no money to pay for the medical consumption will have a significantly lower medical expenses in the month before death.

Conclusions The article explores the importance of constructing effective health management policies and medical consumption guidance from two aspects of health value concept and value medical treatment, in order to promote people to form better medical consumption habits and health management concepts .

Keywords: Disease; Life Course; Traditional Culture Environment; Policy Protection; Value Medicine

\section{Introduction}

Since the 1970s, the acquisition, cost and quality of medical services have become the core goals of medical security in the world. The goal of the global medical reform is to obtain the maximum value of medical care with the minimum medical cost. The key lies not only in medical cost, but also in medical value, namely Value-based Health Care (VBHC) [1].Porter proposed value health care in 2006 . Its core is to create value for patients, integrate medical practices, and measure medical effect and cost. Porter (2010) emphasized the efficacy output of unit medical inputs[2].In 2016, the Chinese government, the World Bank, and the World Health Organization jointly issued a medical reform report_- "Deepening China's Medical and Health System Reform-Building a Value-Based Quality Service Delivery System”, which formally put forward the concept of "Value Medicine". In 2017, the first China Value Medicine Summit Forum put forward a Chinese version of the "values" including efficacy, efficiency, effectiveness, empowerment, and empathy. "Medical 5E Framework "[3]. 
In China, individuals or families are faced with the dilemma of "the law of disease occurrence and the law of life course". Specifically, it is expressed as "should be out-patient or out-patient or should not be hospitalized"; "huge medical expenses before death" and so on. In the process of the change of medical concept, the traditional culture environment and policy changes under the medical security system play an important role. In terms of culture, what are the "normal" expectation for disease and death treatment under the constraints of tradition? In traditional culture environment, the Chinese people's understanding of disease has always been "taboo and disease," while the treatment of death is "fear." With the development of modern medical security system, scholars have not paid enough attention to the evolution direction and trend of traditional medical treatment model and value medical concept . The core of anticipating diseases and death is to establish scientific values of health and life and death. The key lies in how to define norms and values that are consistent with the identity of the patient (or the deceased). In terms of policy protection, medical technology advancement and income growth as well as the gradual reform and improvement of the medical security system, it has promoted the increase of medical demand and costs. With the implementation of the "Healthy China" Strategy, value me dicine will also become an important part of the healthy development of society. However, based on the current health awareness of Chinese residents and the development of China's medical security system, there are still many problems in the development of value medical care. It is worth further studying what role the disease awareness in traditional culture environment and the medical security system of service policy play in the development of value medical care.

\section{Literature review}

From the perspective of traditional culture environment and policy protection under the medical security system, this paper reveals the development path of value-based medical care in China and explores the future development direction. First of all, it is necessary to make clear the logical relationship between policy protection and value medical care under traditional culture environment and medical security system. Secondly, it discusses the inherent transmission mechanism between traditional culture environment, policy protection and value medical care.

\subsection{Traditional Culture Environment: Formation of Value Medical Concept}

Under the influence of traditional culture environment, the traditional notion of "taboo 
disease but not cure it" has deep-rooted influence. Due to the existence of medical risks, there were "medicine donation" activities in ancient China, such as "Kangzi feeding medicine" [4]. During the period of the Republic of China [5], the ideological thread and medical practice of the medical elite have a far-reaching influence on the formation of current medical concepts. With the replacement and reform of medical concept and medical culture, as well as the introduction of Western medicine, there are complex contradictions between the traditional patients who have "acquired the illness for a long time" and "modern patients." Due to the lack of medical common sense, patients are often treated improperly. With the development of medical science, individuals or families have gradually formed the concept that "all diseases have medicine, and no ghosts don't die", placing more responsibility on doctors or the hospital[6]. At the same time, after the entry of western medicine, two sets of medical systems coexisted with the local medical concepts, but the two were completely different in terms of goals, values, knowledge and technology.The process of "looking, hearing, asking and cutting" in traditional medicine is a process of understanding and communicating with patients, listening to them and comforting them[7]. In contrast, modern medicine focuses on the pursuit of the value of scientific medicine itself, separating and analyzing individuals. The changes of modern medical culture still has far-reaching impact on Chinese people's value medical concept.

\subsubsection{The Emergence of Medical Values in Traditional Culture Environment}

Before and after the emergence of scientific medicine and becoming mainstream medicine in modern society, folk prescriptions were not uncommon among ordinary people, especially the lower class with low socioeconomic status and difficult access to formal medical services. In the traditional Chinese family structure, the five basic situations of "home", "raising", "illness", "mourning", and "sacrifice" cover the main content of each stage of parent-child relationship[8]. Among them, "illness" has an important status. "Twenty-four filial piety" describes the behaviour of patients, families and doctors, and discusses behaviors related to diseases. In the pre-industrial society, due to the lack of good education and the lack of access to modern orthodox medical knowledge, Chinese people were more affected by non-professional knowledge in their disease-related behaviors.The "Twenty-Four Filial Piety" involves more patients and family members, and the role of doctors is rarely reflected. Even if there is a relationship with doctors, it is very different from today's doctors. Secondly, the authority of doctors has been challenged by various factors such as medical standards, religious beliefs, popular medical concepts, and folk therapies [9]".The types of medical 
diagnosis and treatment that people choose are closely related to their economic living standards and modern medical and health systems, and is also affected by their own understanding of the causes of the diseases[10] . For example, there still exist local gods and religious beliefs in traditional Chinese society or in some folk areas. For example, under the concept of folk medicine of Baima Tibetan folklore in China, there is a medical practice of "two solutions of magic medicine", that is, taking medicine after getting sick, and believing in God. The gods play two roles, which can cause and cure diseases[11].Under the influence of supernatural forces, that is the cause of the disease and the method to eliminate the disease. Whenever a mass catastrophe endangers the entire society, it is resolved by the means of "Nana Sadu Pearl"[11]. In the practice of folk medicine, due to the mutual relationship among economy, religious beliefs, and inter-ethnic interactions, herbal therapies such as "blood gas", "activity", "eucommiaulmoides", propolis and gastrodia also play an important cultural role.

2.1.2. The Formation of a Value Medical Concept Based on the Development of "Health" and "Disease"

Reasonable definition of "health" and "disease" is an important basis for establishing the scientific values of medicine. The World Health Organization (1978) defines health as health including physical, mental, and social health[12]. From the perspective of dialectical medicine, health is the acquisition and control of the basic material and non-material resources to maintain and promote life with high satisfaction. Among them, unequal social status, class, and social diseases are the important factors that hinder health[13]. Diseases correspond to health, in general, and people still suffer from disease even in the best environmental conditions[14]. As a medical disease, it mainly refers to the direct result of pathogens or physiological disorders. But just like the definition of health, ignoring the social background of environment, occupation, and residence will weaken the social connection of healthy .The social nature of the disease is that it is affected by social factors such as malnutrition, lack of economic security, housing security, and political rights, and it is highly susceptible to disease[15,16]. For example, existing studies have found that productive activities, differences in organizational forms of resources and production, implementation methods, and resource allocation have led to different disease phenomena in different societies[17]. Therefore, diseases have both biological and social characteristics[16]. Based on the reasonable definition of health and disease, a healthy lifestyle can also be defined as a comprehensive standard of life including health care, medical care and health undertakings. 
The medical concept formed on this basis is a scientific medical value.

The modern doctor-patient relationship based on individualism does not exist in traditional Chinese society. For ordinary people, there is no universal cognitive basis for the concept of patient self-responsibility or doctor dependence[18]. A large number of existing studies have shown that under the traditional concept of value medicine, a large proportion of the Chinese population, especially the elderly, are taboo against the disease. On the one hand, they are unwilling to choose to seek medical treatment in time, so at the end of their lives, there is a large amount of medical capital invested in excessive treatment.

\subsection{Policy Protection: the Development and Change of Value Medical Concept}

Not only is the concept of value medical care affected by traditional culture environment, but also the policy protection will play an important role with the development of society and economy. Studies have found that medical needs which are based on self-assessed health[19], chronic disease[20], and restricted mobility[21] are the core indicators affecting individual medical behavior. The health insurance coverage and the soundness of public infrastructure services can help improve individual medical treatment[22]. Characteristic variables such as gender, age, waiting time, distance to medical treatment, and disease severity are also significant influencing factors[23,24,25,26], but the supplier-induced demand factor has a significant impact on the choice of medical treatment for patients who haven't received treatment for the first time[27]. Generally, there are three processes to transform a patient's concept of medical treatment into behavior : first, the decision of the first consultation after the perception of illness, that is, the choice between self-diagnosis and regular diagnosis and treatment; second, the choice of medical institutions; third, the decision on the amount of medical consumption. The three processes always follow the concept of value medical, but will be significantly affected by policy protection. In the $1980 \mathrm{~s}$, China has made great achievements in the field of medical services, but its development in recent decades has not been satisfactory. According to the World Bank (2005), the lack of timely service for patients who require medical services was one of the important reasons[28].

\subsubsection{Research on the Development of Medical Behavior under the Policy Protection Income}

The transmission path of policy protection to the value medical concept is dominated by the economic income guarantee. The family income level has two effects. The first one is the direct effect, that is, the income level increases the health demand, and then increases the level of medical expenditure. For example, if the level of income has a direct impact on the 
behavior of the doctors, "self-treatment" and "treating diseases as secondary" account for a large proportion among the non-patients. The second is the indirect effect, also known as the health effect. Because of the poor health condition of low-income people, they are more likely to get sick[29]. Therefore, a larger amount of medical expenditure is needed to avoid health risks. From the perspective of social psychology, the widening income gap will increase the frustration and pressure of low-income people, and cause depression, or some bad behaviors such as smoking and alcohol abuse, which will worsen the individual's health and bring in higher medical cost to avoid health risks[30]. As an important factor restricting the ability of low-income people to pay for medical treatment, the expansion of medical insurance coverage can alleviate the limitation of the payment ability to a certain extent, and the medical treatment of poor patients is also effected by the history of disease, mobility disorders, and self-assessed health. Research by Wagstaff (2008) shows that the policy protection of medical insurance encourages more people to choose higher medical institutions for medical treatment[31]. As the most important external constraint that affects the individual's use of medical and health resources, the role of medical insurance is still controversial. Among them, there are two views on the impact of medical insurance on individual medical decision-making[32,33,34,35]. The core of the expansion is that the reduction of the relative price of medical services has improved the accessibility of medical services. From the perspective of the process, based on the research of the New Rural Cooperative Medical Service in rural China, the new rural cooperative medical service is facing the shrinking of "death". The core of shrinking is that the medical expenses actually borne by families are rising because of the increasing consumption of medical resources $[36,37,38]$.

\subsubsection{Research on the Change of Medical Decision under the Change of Income Gap}

The ability to pay is the direct carrier of economic income to individual medical treatment decisions. However, some studies have found that group differences of the ability to pay also affect the medical concept of different groups, that is, the income gap has a greater impact. Studies based on micro data have found that there is a strong negative correlation between income gap and health in the United States, which has led to differences in medical perceptions among different groups[39,40]. Other studies have shown that there is no relationship between the two[41]. The income gap will affect public investment and expenditure in health care. The deep manifestation is the differentiation of the interests between the poor and the rich. The rich relatively prefer non-local medical services making 
the supply of local public medical services underestimated and reducing fiscal expenditures[42,43]. This trend will also reduce the effectiveness of the implementation of medical policies,and these policies will be passed health status on to individuals through community basic medical facilities[40,44,45]. However, some studies have shown that constraints such as lower income, lack of medical insurance, and a poor living environment are the core of the gap in wealth between rich and poor, rather than preferences $[36,46]$. The income gap can improve the level of people's health and promote the value of higher medical consumption. For example, the wealthiest group promotes the technology and equipment upgrading of medical institutions in accordance with their own medical needs. Through spillover effects, the overall level of social medical consumption has been improved and people's health has also been improved.Secondly, the income gap drives the growth of fiscal revenue, and the fiscal revenue is converted into fiscal investment in public health and basic medical care, which improves the overall health level of the society and promotes the formation of a more scientific value of medical concept[47,48], as well as comprehensively improves the health level of social development[49,50]. In China, regardless of the size of the disease, people generally prefer to go to a large medical institution where there are more advanced equipment and services. The two-way referral system of "minor illness to community and major illness to hospital", that is, the graded diagnosis and treatment system, which is implemented by the Ministry of Health of the People's Republic of China, has not significantly improved this situation. At present, the imbalance in the use of medical resources remains still serious.

\subsection{Summary of Existing Research and Innovation of this Article}

On the basis of the existing research, we find that the existing research on the formation of the value medical concept under the influence of traditional culture environment has a rich literature basis, while the research on the change of medical values under the protection of policy is more embedded in medical behavior and medical decision-making. Among them, the concept of value medical care is rarely mentioned directly. Even if there are some of them involving value medical care, it can also be viewed from the perspective of the innovation in payment methods of medical insurance policies. 1) From the perspective of research, whether it is to explore the value medical concept from the perspective of traditional culture environment or from the perspective of policy protection, the current research is too much from the perspective of phenomenon and surface [51], ignoring the formation mechanism of the internal values behind individual medical decision-making. 2) In terms of research 
methods, the influence of cultural tradition environments on medical decision-making and behaviors is mostly discussed from a qualitative perspective. In terms of policy protection, the core is to discuss the transfer of income from medical insurance policies, which will cause changes in medical behaviors, but the lack of policy protection deeply reveals the concept of value medicine. 3) As far as the research conclusion is concerned, the existing research, which is about the direction of the influence of cultural tradition environments and policy protection on individual or family medical behavior, has not reached a unified conclusion. The reason lies in excessive dispersion studies of the influence of a certain phenomenon or an insurance policy on medical decisions or behaviors [52,53]. It is the core to study the influence of the formation and change of medical values on individual medical treatment behavior. Therefore, in response to the main defects of the existing research, this article attempts to make breakthroughs and innovations in the following aspects : 1) From the perspective of research, this paper try to explore the formation and change of Chinese people's value medical concept from the perspective of the combination of cultural tradition environment and policy protection. 2) In terms of research methods, through the combination of qualitative and quantitative research, this paper not only analyzes the core concepts of value medical concepts, such as disease and health positioning, but also empirically tests the formation and change of the influence path of traditional culture environment and policy protection on Chinese people's concepts of value medicine over the decades. 3) In terms of research content, this paper focuses on the occurrence of diseases and the laws of life processes, and highlights the analysis of the formation and changes of the value medical concept, in order to enrich the research content of the value medical concept. 4) On the conclusion of the study, the reform and optimization of the payment system of medical expenses are put forward by carding and empirical testing of the development and changes of medical value concept in China.

\section{Research data and descriptive statistics}

\subsection{Data Sources}

The data of this article are selected from the survey data of the 2014 Chinese Longitudinal Healthy Longevity Survey (CLHLS). The survey scope of the CLHLS database covers 23 provinces, autonomous regions and municipalities in the country. The subjects of the survey were mainly elderly people aged 65 and above and their adult children aged 35 to 64 . The 
questionnaire is divided into two parts: the questionnaires for the family member of the dead and the questionnaires for living respondents. In this study, a sample of survivors was selected to investigate the medical values and behaviors of residents in the treatment of disease. A sample of dead elderly was used to investigate the medical values and behaviors in the course of life. According to the characteristics of CLHLS's continuous follow-up survey sample, taking 1998 as the baseline, the cross-sectional data from 1998 to 2014 in this article were selected to construct an unbalanced panel, and 6979 valid samples were obtained. In addition, this article also selects data from the analysis report of the National Health Service Survey, which is surveyed every five years from 1993 to 2013, and the changes of medical consumption habits of urban and rural residents are statistically analyzed.

\subsection{Core Variable Selection and Descriptive Statistics}

\subsubsection{Core Variable Selection}

The changes of value medical concept under the protection of cultural tradition environments and cultural policies are affected not only by subjective factors but also by corresponding objective factors. This article attempts to discuss the changes of Chinese residents' medical values from the perspectives of the law of disease occurrence and the law of life course.

First of all, in terms of the occurrence of diseases, the outpatient who should receive out-patient service but not and inpatient who should be hospitalized but not were selected as the core variables to be explained, and the influence of variables such as health status, income changes, and medical insurance policy interventions on individual medical behaviors was examined. The health status is reflected by the current health status in the questionnaire, and the health status in the statistical items, which is very good or well is defined as health and recorded as 1; health status which is biased and very poor is defined as unhealthy and recorded as 0 . Per capita income of the households is used as the income variable, while that basic medical insurance chooses to participate in basic medical insurance is used as the proxy variable, that is, if residents participates in New Rural Cooperative Medical Care, Urban Residents Medical Insurance, Urban Workers Medical Insurance, Urban and Rural Resident Medical Insurance or Public Medical Care, it is recorded as 1, otherwise 0 .Secondly, in terms of the law of life course, we take the medical consumption expenditure of the elderly before their death and the place of death as the explanatory variables to examine the impact of income growth and policy intervention on the medical consumption of end-of-life patients by using income and the burden of medical consumption before death as core explanatory 
variables.

According to the previous analysis and the definition of core variables, the article attempts to select the basic model settings, and plans to use the binary Probit model to test the outpatient and non-inpatient rates; the mixed Tobit model is used to test the elderly end-of-hospital medical expenditure and the factors that affect the location .

\subsubsection{Descriptive Statistics}

The descriptive statistics of the core variables in the article are shown in Table 1. The proportion of outpatients for urban and rural residents was $77.55 \%$, and the proportion of inpatients was $41.84 \%$. The proportion of people with basic medical insurance is $92.13 \%$, the proportion of dying in hospitals is $10.49 \%$, and the proportion of the bedridden before dying is $70.47 \%$. The definitions and descriptive statistics of other variables are shown in Table 1 .

Table 1 Descriptive statistics of core variables

\begin{tabular}{|c|c|c|c|c|c|}
\hline Variable & Variable definitions & Mean & S.D & Min & Max \\
\hline Whether outpatient & Outpatient $=1$, No outpatient $=0$ & 0.7755 & 0.42 & 0 & 1 \\
\hline Whether to be hospitalized & Has hospitalization spending $=1, \mathrm{no}=0$ & 0.4184 & 0.49 & 0 & 1 \\
\hline Health & $\begin{array}{l}\text { The self-assessed health is very good or better }=1 \text {, the } \\
\text { others }=0\end{array}$ & 0.4061 & 0.49 & 0 & 1 \\
\hline Unhealthy & the self-assessed health is poor $=1$,others $=0$ & 0.2263 & 0.42 & 0 & 1 \\
\hline $\begin{array}{l}\text { Participate in Basic Medical } \\
\text { Insurance }\end{array}$ & $\begin{array}{l}\text { Participation in in Basic Medical Insurance }=1 \text {, } \\
\text { others }=0\end{array}$ & 0.9213 & 0.27 & 0 & 1 \\
\hline LnIncome & Log of household per capita income & 8.1446 & 1.54 & 0 & 11.51 \\
\hline Urban and rural & Rural $=1$, Urban $=0$ & 0.5541 & 0.50 & 0 & 1 \\
\hline Severe physical restriction & Severe physical restriction $=1$, others $=0$ & 0.1269 & 0.33 & 0 & 1 \\
\hline Physically restricted & Physically restricted $=1$, others $=0$ & 0.2194 & 0.41 & 0 & 1 \\
\hline Completely unrestricted & Completely unrestricted $=1$, others $=0$ & 0.6512 & 0.48 & 0 & 1 \\
\hline Lnmedical_cost & Logarithmic value of dying medical cost & 7.0842 & 2.00 & 0 & 11.51 \\
\hline Dying in hospital & Dying in hospital $=1$, others $=0$ & 0.1049 & 0.31 & 0 & 1 \\
\hline Number of serious injuries & $\begin{array}{l}\text { Number of serious injuries requiring hospitalization } \\
\text { or bedridden compared to the previous survey }\end{array}$ & 0.4711 & 1.03 & 0 & 40 \\
\hline Bedridden & Bedridden before death $=1$, others $=0$ & 0.7047 & 0.46 & 0 & 1 \\
\hline
\end{tabular}

Secondly, according to the data of five surveys from 1993 to 2013 conducted by the survey 
group of China Health Service, they made statistics on the proportion of the Chinese urban and rural families who should be out-patient but not out-patient, who should be hospitalized but not hospitalized, and why they should be hospitalized but not hospitalized from 1993 to 2013. The results are shown in Table 2. From 1993 to 2013, the rate of non-outpatient who should be out-patient increased from $36.4 \%$ in 1993 to $48.9 \%$ in 2003 , but gradually decreased after 2008. The reason was that the pilots of the new rural cooperative medical system (NRCMS) has played an important role since 2003. The increase rate of non-outpatient service in rural area was higher than that of urban residents. In terms of hospitalization, the overall rate of non-hospitalization is decreasing, from 35.9\% in 1993 to $17.1 \%$ in 2013 , of which the decline in rural areas is much higher than that in urban areas. In terms of the inpatient behavior, the self-recognition of mild illness and financial difficulties which reflect the value of the medical concept have always been the most important restrictive factors. In 2013, the statistical caliber was biased and ignored.

Table 2 Changes in the medical behavior of the Chinese population under five surveys from 1993 to 2013

\begin{tabular}{|c|c|c|c|c|c|c|c|c|c|}
\hline \multirow[b]{2}{*}{ Year } & \multirow[b]{2}{*}{ Classification } & \multirow[b]{2}{*}{$\begin{array}{l}\text { Should be } \\
\text { out-patient } \\
\text { rate. (\%) }\end{array}$} & \multirow{2}{*}{$\begin{array}{l}\text { Should be } \\
\text { hospitalized } \\
\text { without } \\
\text { hospitalization } \\
\text { rate. }(\%)\end{array}$} & \multicolumn{6}{|c|}{ Reasons for non-hospitalization rate } \\
\hline & & & & $\begin{array}{l}\text { Personally } \\
\text { think that the } \\
\text { illness is } \\
\text { mild }(\%)\end{array}$ & $\begin{array}{l}\text { No } \\
\text { time } \\
(\%)\end{array}$ & $\begin{array}{c}\text { Economic } \\
\text { difficulties } \\
(\%)\end{array}$ & $\begin{array}{l}\text { Poor } \\
\text { service } \\
(\%)\end{array}$ & $\begin{array}{l}\text { No } \\
\text { bed } \\
(\%)\end{array}$ & $\begin{array}{c}\text { other } \\
(\%)\end{array}$ \\
\hline \multirow{4}{*}{1993} & total & 36.4 & 35.9 & 16.39 & 12.18 & 55.97 & 1.09 & 4.08 & 10.29 \\
\hline & urban & 42.4 & 26.18 & 18.09 & 15.07 & 40.96 & 2.13 & 10.46 & 13.3 \\
\hline & rural & 33.7 & 40.59 & 15.86 & 11.29 & 60.63 & 0.77 & 2.09 & 9.36 \\
\hline & total & 38.45 & 32.3 & 20.16 & 5.26 & 62.71 & 0.41 & 0.35 & 11.1 \\
\hline \multirow[t]{3}{*}{1998} & urban & 49.56 & 27.5 & 20.77 & 4.34 & 60.09 & 0.35 & 0.94 & 13.5 \\
\hline & rural & 33.26 & 34.5 & 19.93 & 5.61 & 63.69 & 0.44 & 0.13 & 10.21 \\
\hline & total & 48.9 & 29.6 & 19.5 & 4.1 & 70.0 & 0.3 & 0.4 & 5.7 \\
\hline \multirow[t]{3}{*}{2003} & urban & 57.0 & 27.8 & 30.7 & 3.2 & 56.1 & 0.8 & 1.0 & 8.2 \\
\hline & rural & 45.8 & 30.3 & 15.1 & 4.4 & 75.4 & 0.1 & 0.2 & 4.8 \\
\hline & total & 37.6 & 25.1 & 10.7 & 7.7 & 70.3 & 0.1 & 0.8 & 10.4 \\
\hline \multirow[t]{2}{*}{2008} & urban & 37.3 & 26.0 & 13.9 & 4.5 & 67.5 & 0.1 & 1.3 & 12.8 \\
\hline & rural & 37.8 & 24.7 & 9.5 & 9.0 & 71.4 & 0.1 & 0.6 & 9.5 \\
\hline \multirow{2}{*}{2013} & total & 27.3 & 17.1 & 4.1 & 2.0 & 7.4 & - & 0.3 & 3.4 \\
\hline & urban & 32.9 & 17.6 & 4.4 & 1.7 & 7.2 & - & 0.4 & 3.8 \\
\hline
\end{tabular}


Note: The data are from the five survey data of the National Health Service Survey and Analysis Report from 1993 to 2013.

\section{Empirical test}

\subsection{Disrespect for the Pattern of Disease}

4.1.1Health Status and non-attendance rate of Outpatient serviced in Urban and Rural Areas

It is an important aspect to reflect the traditional medical concept of "taboo disease and taboo medicine" that the patients who should be outpatient are not outpatient and the patients who should be hospitalized are not inpatient. Here we also try to control the income and the intervene of medical insurance policy to test the actual basis for not respecting the laws of disease's occurrence. The test results are shown in Table 3 , in which models (1), (2), and (3) are tested with rural samples. The results show that under model (1), health significantly reduces the probability of outpatient service, while being unhealthy significantly increases the probability of outpatients. Income is a significant positive factor to improve the tendency of outpatient service of rural residents, while basic medical insurance has no significant effect. After controlling the interaction between health and participation in basic medical insurance, the test results of the model (2) show that health significantly reduces the outpatient probability of rural residents who enjoy the basic medical insurance. Considering the endogeneity of the results, the article examines and deals with the endogeneity of health and outpatient clinics, and selects the degree of residents' physical restriction as a tool variable. The results of endogenous Wald test show that endogeneity exists and tool variable processing is effective. After endogenous treatment, health is still significantly negative, but unhealthy is no longer significant, and unhealthy is also a negative effect, that is, an individual's unhealth negatively reduces the rate of outpatient service, that is, the cultural concept in theory affects the phenomenon that residents should attend outpatient service but not. Models (4), (5), and (6) based on urban samples show that health also has a significant negative effect on the outpatient probability of urban residents, but the unhealthy has no significant effect. Model (5) shows that the interaction between health and basic medical insurance has no significant effect. However, the results of model (6) with instrumental variables show that both health and ill health significantly reduce the outpatient probability of urban residents, and there is no significant impact on income and basic medical insurance, that is, whether residents are healthy or not, urban residents show a lower outpatient 
tendency.The reason is that due to the influence of traditional culture environment, when an individual is healthy, there are fewer outpatient choices, and when he is unhealthy, due to the concept of "taboo disease and avoid medical treatment", his choice of outpatient rate is still low, so there is a significant negative impact. The rate of outpatient who shoulds be out patient but not is significantly higher.

Table 3 Urban and rural residents' health status, basic medical insurance, income and outpatient rate

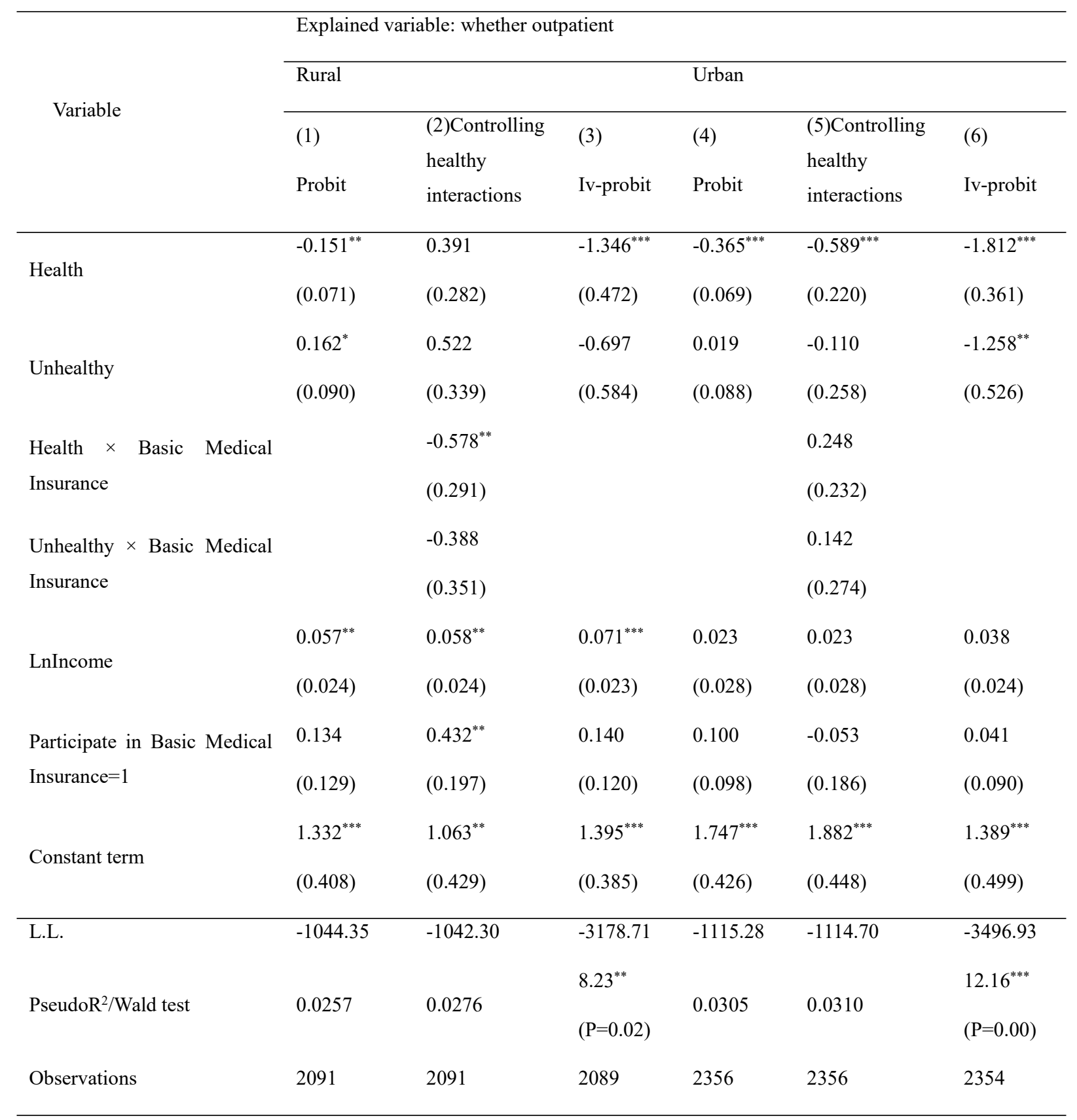

Note: Standard errors are in parentheses, $* \mathrm{p}<0.1, * * \mathrm{p}<0.05$, and $* * * \mathrm{p}<0.01$. Other control variables are omitted. 


\subsubsection{Health Status and Urban and Rural should be Hospitalized But Not Hospitalized}

Generally speaking, outpatient clinics is a response to the general illness or chronic illnesses of the residents, while inpatient clinics is a response to more severe illnesses of the residents. Therefore, this paper conducts an empirical test on the health status of urban and rural residents and the situation of residents who should be hospitalized and but not. The results are shown in Table 4. The test results of models (1), (2), and (3) using rural samples show that health significantly reduces the probability of hospitalization for rural residents, while ill health significantly increases the probability of hospitalization for rural residents. The interaction between health and basic medical insurance has a negative effect, that is, health weakens the positive effect of basic medical insurance on hospitalization. However, the results of the treatment of tool variables in model (3) show that neither health nor ill health has a significant effect, and the significant positive effect of income level does not increase the rate of inpatient, that is, factors other than non-economic factors, especially cultural factors still play a potential role, which directly weakens the positive effect of income growth and health insurance policies. Models (4), (5), and (6) based on urban samples all show that health has a significant negative effect on the hospitalization rate of urban residents, and the coefficients becomes larger after the treatment of the instrumental variables. At the same time, ill health also has significantly negative effect on urban residents, that is, as for the patient who should be hospitalized but not, urban residents also show a lower hospitalization rate, but cultural tradition environments have a far greater impact on hospitalization rates of urban residents than that of rural residents.

In conclusion, from the two aspects of non-outpatient service and non-inpatient service , cultural tradition environments have a greater impact on the medical value of urban residents than that of rural residents. Affected by traditional culture environment, urban residents used to disregard the law of the disease occurrence. With the increase of basic medical insurance and income, they now attach importance to prevention, but have not formed better medical values. The phenomenon of "taboo disease and taboo medical treatment" is still serious, that is, the health variables in the test results have a significant negative effect on their outpatient service or inpatient service. However, the medical values of rural residents have not changed significantly. This conclusion is verified by the result that the unhealthy factors have not significantly affected their outpatient or hospitalization. 
Table 4 Urban and rural residents' health status, basic medical insurance, income and hospitalization rate

\begin{tabular}{|c|c|c|c|c|c|c|}
\hline \multirow{4}{*}{ Variable } & \multicolumn{6}{|c|}{ Explained variable: whether hospitalized } \\
\hline & \multicolumn{3}{|l|}{ Rural } & \multicolumn{3}{|l|}{ Urban } \\
\hline & (1) & $\begin{array}{l}\text { (2)Controlling } \\
\text { healthy }\end{array}$ & (3) & (4) & $\begin{array}{l}\text { (5)Controlling } \\
\text { healthy }\end{array}$ & $(6)$ \\
\hline & Probit & interactions & Iv-probit & Probit & interactions & Iv-probit \\
\hline \multirow{2}{*}{ Health } & $-0.224^{* * *}$ & 0.341 & -0.612 & $-0.456^{* * *}$ & $-0.724^{* * *}$ & $-2.086^{* * *}$ \\
\hline & $(0.065)$ & $(0.268)$ & $(0.629)$ & $(0.061)$ & $(0.196)$ & $(0.184)$ \\
\hline \multirow{2}{*}{ Unhealthy } & $0.134^{*}$ & 0.337 & 0.196 & 0.105 & -0.065 & $-1.139^{* *}$ \\
\hline & $(0.076)$ & $(0.303)$ & $(0.651)$ & $(0.072)$ & $(0.213)$ & $(0.448)$ \\
\hline \multirow{2}{*}{$\begin{array}{l}\text { Health } \times \text { Basic Medical } \\
\text { Insurance }\end{array}$} & \multicolumn{3}{|c|}{$-0.600^{* *}$} & \multicolumn{3}{|c|}{0.297} \\
\hline & \multicolumn{3}{|c|}{$(0.275)$} & \multicolumn{3}{|c|}{$(0.206)$} \\
\hline \multirow{2}{*}{$\begin{array}{l}\text { Unhealthy } \times \text { Basic } \\
\text { Medical Insurance }\end{array}$} & \multicolumn{3}{|c|}{-0.216} & \multicolumn{3}{|c|}{0.189} \\
\hline & & $(0.313)$ & & & $(0.225)$ & \\
\hline \multirow{2}{*}{ LnIncome } & $0.055^{* *}$ & $0.056^{* *}$ & $0.066^{* * *}$ & $0.051^{* *}$ & $0.052^{* *}$ & $0.061^{* * *}$ \\
\hline & $(0.022)$ & $(0.022)$ & $(0.023)$ & $(0.024)$ & $(0.024)$ & $(0.023)$ \\
\hline \multirow{2}{*}{$\begin{array}{l}\text { Participate in } \text { Basic } \\
\text { Medical Insurance }=1\end{array}$} & 0.001 & 0.278 & 0.013 & -0.017 & -0.181 & -0.043 \\
\hline & $(0.120)$ & $(0.196)$ & $(0.119)$ & $(0.088)$ & $(0.149)$ & $(0.076)$ \\
\hline \multirow{2}{*}{ Constant term } & 0.513 & 0.251 & 0.573 & -0.142 & 0.002 & 0.128 \\
\hline & $(0.366)$ & $(0.395)$ & $(0.385)$ & $(0.374)$ & $(0.388)$ & $(0.323)$ \\
\hline L.L. & -1344.62 & -1342.19 & -3478.32 & -1521.18 & -1520.12 & -3880.19 \\
\hline PseudoR²/ & \multirow[b]{2}{*}{0.040} & \multirow[b]{2}{*}{0.042} & $6.48^{* *}$ & \multirow[b]{2}{*}{0.039} & \multirow[b]{2}{*}{0.039} & $75.70^{* * *}$ \\
\hline Wald test & & & $(\mathrm{P}=0.04)$ & & & $(\mathrm{P}=0.00)$ \\
\hline Observations & 2091 & 2091 & 2089 & 2356 & 2356 & 2354 \\
\hline
\end{tabular}

Note: Standard errors are in parentheses, ${ }^{*} \mathrm{p}<0.1,{ }^{* *} \mathrm{p}<0.05$, and ${ }^{* * *} \mathrm{p}<0.01$. Other control variables are omitted. Here, the instrumental variables are still selected as physical restricted variables, and the wald test shows that they are endogenous and the treatment is effective.

\subsection{Disrespect for the Laws of Life Course}

\subsubsection{Changes in the Final Place of the Elderly and the Quality of Life}

The other aspect of the value medical concept is the impact of policy intervention, especially after the implementation of the basic medical insurance policy, the cost compensation 
mechanism has improved the traditional medical concept, and many scholars have also carried out researches. At the same time, after 2000, with the gradual increase in income levels, the Chinese people's concept of medical treatment has also changed greatly. This change includes not only the high elasticity of medical consumption under income growth but also the moral hazard generated by the design of the basic medical insurance system. But throughout it all, there is still the reaction to the laws of life course. In the early days, the Chinese people's medical concept was more constrained by tradition or economy, that is, limited economic capabilities. After entering the new period, this phenomenon has improved, but it showed excessive medical treatment, At the end of life, because of the lack of death education and other Multiple reasons, most people still invest a lot of money in treatment, resulting in a waste of medical resources. In this paper we attempt to analyze the elderly hospice location and the quality of life before death.

As is shown in Tables 5 and 6, the results of the CLHLS longitudinal survey collects the old adults' location for death and their quality of life before the death from 2000 to 2014. In the survey, the dying places of the elderly mainly includes homes, medical institutions, old-age care institutions, or other institutions, among which, medical institutions mainly refer to the samples of the elderly who died in the hospital.The results in Table 5 show that from the six follow-up surveys from 2000 to 2014, the sample proportion of home deaths decreased from $90.10 \%$ to $81.08 \%$, which is decreasing on the whole, but still high, indicating that most elderly people still die at home. However, the proportion of death samples in medical institutions increased from $5.66 \%$ to $12.16 \%$, showing a clear upward trend. Especially after 2005 , the reason why the increase is so great is that during this period, basic medical insurance was gradually implemented in urban and rural China and household per capita income was increasing rapidly. The sample of deaths in nursing homes or other places did not show a significant increase or decrease. For example, the proportion of deaths in nursing homes increased from 2000 to 2011, but declined significantly in 2014.

Table 5 Changes in the final place of the elderly

\begin{tabular}{|c|c|c|c|c|c|c|c|c|}
\hline \multirow{4}{*}{$\begin{array}{l}\text { Yea } \\
\mathbf{r}\end{array}$} & \multicolumn{8}{|c|}{ Place of death } \\
\hline & \multicolumn{2}{|l|}{ Home } & \multicolumn{2}{|l|}{ Hospital } & \multicolumn{2}{|c|}{ Institution } & \multicolumn{2}{|l|}{ Other } \\
\hline & Statistic & Percentage( & Statistic & Percentage( & Statistic & Percentage( & Statistic & Percentage( \\
\hline & $\mathbf{s}$ & $\%)$ & $\mathbf{s}$ & $\%)$ & $\mathbf{s}$ & $\%)$ & $\mathbf{s}$ & $\%)$ \\
\hline $\begin{array}{l}200 \\
0\end{array}$ & 3011 & 90.10 & 189 & 5.66 & 114 & 3.41 & 28 & 0.83 \\
\hline
\end{tabular}




\begin{tabular}{|c|c|c|c|c|c|c|c|c|}
\hline $\begin{array}{l}200 \\
2\end{array}$ & 1418 & 88.51 & 106 & 6.62 & 62 & 3.87 & 16 & 1.00 \\
\hline $\begin{array}{l}200 \\
5\end{array}$ & 1136 & 87.05 & 90 & 6.90 & 72 & 5.52 & 7 & 0.53 \\
\hline $\begin{array}{l}200 \\
8\end{array}$ & 416 & 86.67 & 35 & 7.29 & 27 & 5.63 & 2 & 0.41 \\
\hline $\begin{array}{l}201 \\
1\end{array}$ & 140 & 79.55 & 21 & 11.93 & 14 & 7.95 & 1 & 0.57 \\
\hline $\begin{array}{l}201 \\
4\end{array}$ & 60 & 81.08 & 9 & 12.16 & 3 & 4.05 & 2 & 2.70 \\
\hline
\end{tabular}

Note: The data comes from the CLHLS database of families tracking dead people from 2000 to 2014.

Table 6 lists the quality of life of the dying elderly at the end of life. The results show that the number of days of care for the most important caregivers in the last month before death has increased from an average of 13.109 days in 2000 to 23.2733 days in 2011 . The number of days of care for the majority of the elderly before dying is increasing. The elderly received timely treatment before they died. From 2000 to 2008, there was an increase in access to timely end-of-life care for the elderly, but it fell back in 2011, with little overall change. In terms of the total medical expenses of the elderly in the month before their death, the average was 12820.16 yuan in 2000 and 10624.17 yuan in 2011, but there was a large decrease in 2002 and it began to rise in 2005, that is, the basic medical insurance system and income growth effect has begun to appear.

Table 6 Life status of the elderly before death

\begin{tabular}{llll}
\hline \multicolumn{5}{l}{ Year } & \multicolumn{3}{l}{ Statistics of main status before death } \\
\cline { 2 - 4 } & $\begin{array}{l}\text { Days of caregiving provided by the } \\
\text { primary caregiver in the last month of } \\
\text { life }\end{array}$ & $\begin{array}{l}\text { The deceased elder get timely } \\
\text { treatment when he/she was ill } \\
\text { before dying }(\%)\end{array}$ & $\begin{array}{l}\text { Total medical costs of the } \\
\text { deceased elder in the last } \\
\text { year of life }\end{array}$ \\
\hline 2000 & 13.311 & 92.65 & 12820.16 \\
2002 & 12.324 & 93.51 & 7552.649 \\
2005 & 20.184 & 94.11 & 10199.41 \\
2008 & 20.934 & 94.96 & 8992.696 \\
2011 & 23.273 & 92.59 & 10624.17 \\
2014 & 0.000 & 0.00 & 0.00
\end{tabular}

Note: The data comes from the CLHLS database of families tracking elderly people who died from 2000 to 2014, and the sample of relevant statistics in 2014 is accurate. 
4.2.2 Impact of Income and Policy Interventions on Dying Medical Consumption of the Elderly

\subsubsection{Income and Medical Expenditure for the Elderly}

Considering the coexistence of positive continuous distribution and zero consumption of medical expenditure, the article attempts to establish a mixed panel Tobit model for testing. First of all, considering the existence of individual effects, panel Tobit regression with random effects should be used, but the LR test results show that the original hypothesis of " $H_{0}: \sigma_{u}$ " has not been rejected, that is, there is no individual effect, and mixed Tobit regression can be used. The results of model (1) and model (4) in Table 7 show that the income level has significantly increased the level of medical expenditures in the month before the end of life, and significantly increased the probability of death in a medical institution for the elderly, that is, the higher the income level is, the more medical consumption the older people will spend before the end of life; the more likely the elder will be to die in medical institutions. According to the results of Wald's test, it rejects the original hypothesis of exogeneity that there is endogeneity. Therefore, whether the patients is incapacitated or bedridden before death is chosen as the instrumental variable for processing. The test results after processing the instrument variables show that the instrument variables are reasonable and effective. After treatment, the results show that income still has a significant positive effect on the medical consumption of the elderly before the death, and the coefficient value is higher. But it no longer has a significant effect on the place of death for the elderly, indicating that income has no significant effect on the place of death of the elderly.

Table 7 Influencing factors of medical expenses and place of death of the elderly

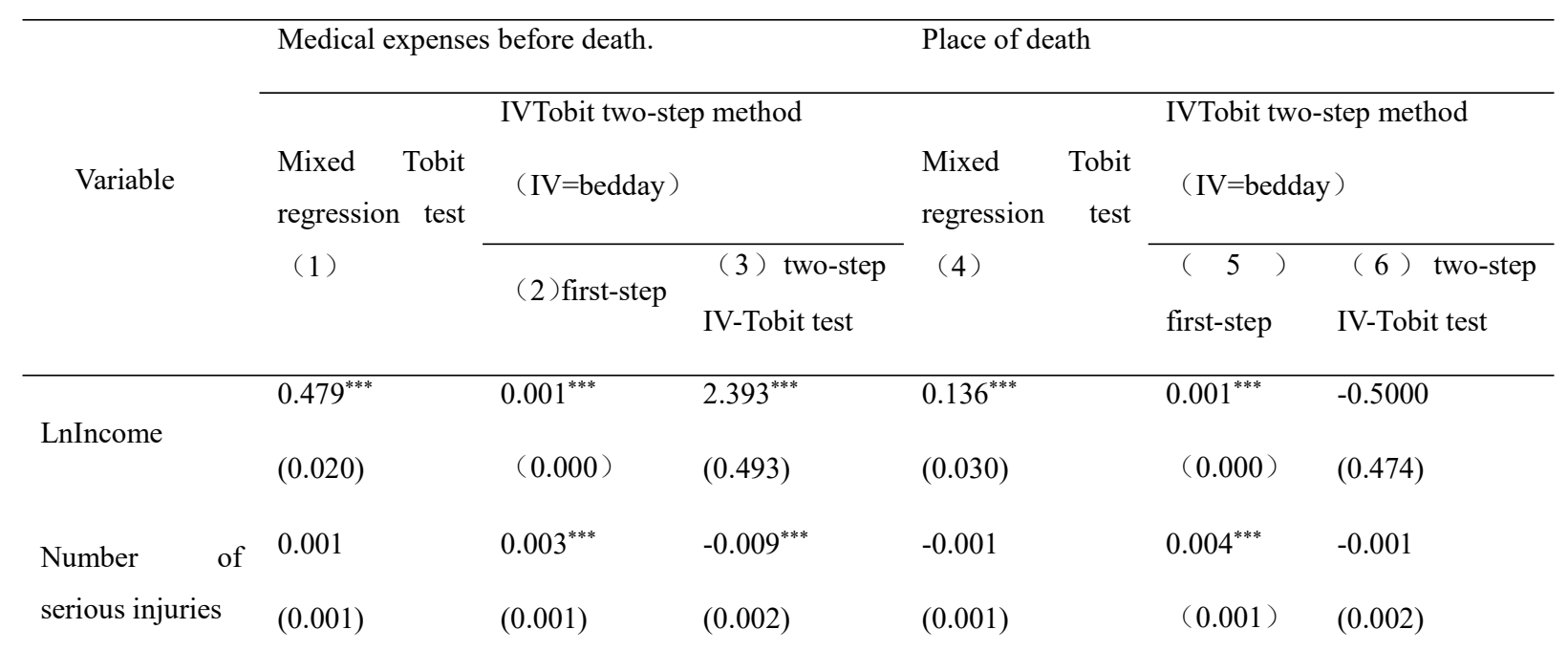




\begin{tabular}{|c|c|c|c|c|c|c|}
\hline Constant term & $\begin{array}{l}6.833^{* * *} \\
(0.468)\end{array}$ & $\begin{array}{l}7.990^{* * *} \\
(0.407)\end{array}$ & $\begin{array}{l}-8.225^{* *} \\
(4.047)\end{array}$ & $\begin{array}{l}1.550^{* *} \\
(0.683)\end{array}$ & $\begin{array}{l}8.0810^{* * *} \\
(0.383)\end{array}$ & $\begin{array}{l}6.578^{*} \\
(3.903)\end{array}$ \\
\hline sigma_u & $\begin{array}{l}0.000 \\
(124.7)\end{array}$ & & & $\begin{array}{l}0.000 \\
(19.37)\end{array}$ & & \\
\hline sigma_e & $\begin{array}{l}1.860^{* * *} \\
(0.022)\end{array}$ & & & $\begin{array}{l}1.805^{* * *} \\
(0.084)\end{array}$ & & \\
\hline $\begin{array}{l}\text { LR test of } \\
\text { sigma_u }=0\end{array}$ & $\begin{array}{l}\operatorname{chibar} 2(01)= \\
0.00(p=1.00)\end{array}$ & & & $\begin{array}{l}\operatorname{chibar} 2(01)=0.00 \\
(p=1.0)\end{array}$ & & \\
\hline Wald test & & $\operatorname{chi} 2(1)=58.51$ & $(\mathrm{P}=0.000)$ & & $\operatorname{chi} 2(1)=2.01$ & $1(\mathrm{P}=0.056)$ \\
\hline Observations & 3627 & 2672 & 2672 & 4378 & 3064 & 3064 \\
\hline
\end{tabular}

Note: Standard errors are in parentheses, ${ }^{*} \mathrm{p}<0.1,{ }^{* *} \mathrm{p}<0.05$, and ${ }^{* * *} \mathrm{p}<0.01$. Other control variables are listed.

4.2.3Family Support, Policy Protection and End-of-life Medical Consumption for the Elderly Second, after controlling the undertaker of the medical consumption before death, the effect of policy intervention on the medical care consumption and location of the elderly before death was investigated. The test results are shown in Table 8. The results of model (1) with medical consumption expenditure as the explanatory variable show that compared with other undertakers, the old people whose medical expenses are borne by the spouse or children living together will have significantly lower medical expenditures at the end of life, that is, the care of a spouse or children living with him has reduced their medical expenses. While the elderly who are subsidized by the state or collective subsidy for medical expenses and the elderly who have no money to pay for medical consumption will have a significantly lower medical expenses. Because this group of people is at a lower income level, and government subsidies cannot increase their high level of medical consumption demand, thus Showing significantly lower end-of-life medical consumption, that is, income is still the core factor that determines the medical consumption of this group, and policy intervention has not effectively improved their medical behavior or medical consumption values. The results of model (2) show that when medical costs are borne by cooperative medical care, the probability of the elderly dying in the hospital will be significantly reduced, that is, the rural elderly are more likely to die at home. Affected by distance or medical costs, the dying state of rural elderly has not been improved by the new Rural Integration. When their medical expenses are borne by the national medical insurance fun, medical insurance,state or collective subsidy medical expenses, these old people who can not afford to pay the medical expenses himself will have significantly higher probability of death at medical institutions. 
Although such old people do not have a higher medical consumption in the last month of their life, they still choose to go to hospital for treatment at the end of their life. It is policy interventions that have improved the higher medical consumption of the elderly in the short term before they die. In other word, the final quality of their life is improved through excessive or invalid medical consumption. On the one hand, there is a lack of good education on the concept of education; On the other hand, there is a large amount of waste of medical resources. Such end-of-life care tends to be more expensive and more resource intensive, but less effective.In addition, the elderly who have no money to pay for medical expenses here are those who have neither insurance nor family support, so they are also groups who in fact enjoy policy-based medical assistance.The high probability of their death at medical institutions indicates that the medical quality of such groups is still relatively poor, and they don't go to the hospital until the minor illness can not be cured, thus increasing the probability of death in the hospital.

Table 8 Impact of policy interventions on end-of-life medical consumption and location of the elderly

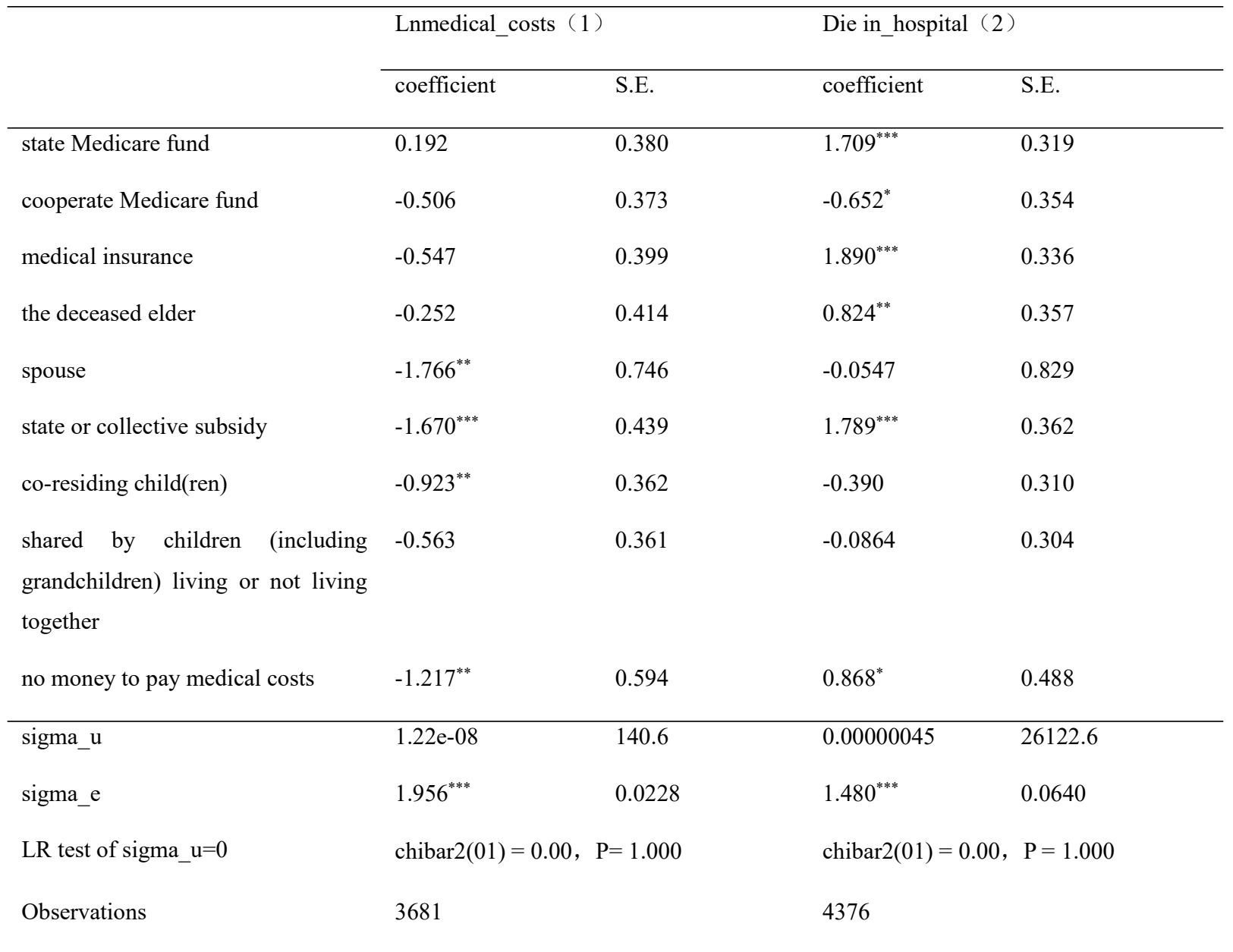

Note: Standard errors are in parentheses, $* \mathrm{p}<0.1, * * \mathrm{p}<0.05$, and ${ }^{* * *} \mathrm{p}<0.01$. The control variables are the same and are omitted here. 


\section{Discussion}

Whether it is based on the law of disease occurrence or the law of life course,and how to build good medical values, we must first make clear what the goal of medical consumption is, to maintain or restore self health? So, what is the definition of health? Is there no disease or is basic physical function recovery? Health, as the World Health Organization defined in 1948, is not only a state of not being sick or weak, but also a state of physical, mental and social integrity. ${ }^{1}$ However, in reality, the traditional health concept which is "equal to not getting sick" or "centering on treating diseases" has almost formed the concept of health value in the specific background[49]. Under the current social concept, the pursuit of health has become alienated and one-sided. Health behaviors are defined as the act of getting sick or getting rid of diseases, while the original intention of medicine is to restore or cure injuries or diseases, which here refer to the injuries and diseases seriously affecting the life function of the patients. Patients are living functions, and not everyone should be included in the medical system. For example, from a perspective of health, people can be divided into three categories: health, sub-health, and unhealthy[50]. Among them, the unhealthy group should be the focus of the current or future medical system, that is, the real object of medical services, Healthy and sub-healthy groups should be maintained or promoted through socialized health management policies, which emphasizes the intervention of the initial end of the health of these groups to improve their health quality.

Based on this, for healthy or sub-healthy people, should we pay more attention to health prevention or after-care medical investment? This is a common problem in the reform of the medical insurance system in various countries at this stage. At the 2016 National Congress of Health and Hygiene, General Secretary Xi Jinping put forward the concept that "we should establish the concept of big hygiene and great health, and transform the focus on treating disease into the focus on people's health."2Due to the gradual increase in the size of such populations, from the perspective of sociology, it is necessary to change the traditional health concept centered on the treatment of diseases and improve the quality of health through effective health management. Here, health management should include macro policy guidance and micro Individual behaviors and habits, such as nutrition interventions, sports interventions, psychological interventions and other policy interventions, as well as micro-management policies such as healthy living habits or methods, and traditional Chinese

\footnotetext{
${ }^{1}$ https://www.who.int/about/who-we-are/constitution.

$2 \mathrm{http}: / /$ www. xinhuanet.com/politics /2016-08/20/c_1119425802.htm.
} 
medicine for disease prevention and treatment to improve the health and quality of such people. Regarding the medical concept of unhealthy people, it is necessary to promote their reasonable medical consumption by means of medical guidance services, to gradually reduce the rate of non-outpatient who should be outpatient through policy interventions such as medical insurance, to avoid the phenomenon of "taboo disease and avoid medical treatment", and to improve rationality and effectiveness of their medical consumption. According to the current law of life course, most of the elderly still have a high medical consumption of end-of-life medical care at the end of their lives, while in terms of the law of disease occurrence, policy intervention has not increased the probability of non-outpatient who should be outpatient. From the implementation effect of the current medical insurance policy, on the one hand, the traditional culture environment of "taboo diseases and taboo medical treatment" still plays a role in core medical values to a certain extent. On the other hand, when minor illnesses which are not cured timely turn to major illnesses or the state of illnesses before dying, there is a phenomenon of excessive medical consumption expenditure.Therefore, to explore medical values based on the concept of health values, we need to pay attention to the effectiveness of policy intervention for the unhealthy people and the guidance of medical consumption for end-of-life patients, that is, early intervention and palliative care for diseases. The policy intervention of disease prevention should not rely too much on basic medical insurance and should be promoted step by step in combination with the implementation of health management policies. When healthy state is reduced to an unhealthy state, timely treatment must be guided by reasonable medical services to enhance the utilization of medical services and reduce resources waste. At the same time, through policy intervention of end-of-life personnel, through medical guidance and service delivery, the rationality of end-of-life medical consumption is promoted and the waste of medical resources are avoided. In order to achieve effective policy connection and protection of the initial, the middle and the terminal of the disease occurrence, it's necessary to promote unhealthy people to form a good value medical concept, and rationalize the consumption of medical resources.

\section{Conclusion}

Based on the law of disease occurrence and the law of life course, this paper uses the data of the "Tracking Survey of the Influencing Factors of Aging Health in China" and "National 
Health Service Survey and Analysis Report" to explore the changes in medical values under the protection of traditional culture environment and policies, which is expected to provide reliable theoretical and empirical support for reasonable medical consumption behavior and health management policies. The research concludes that from 1993 to 2013, the number of non-outpatient who should be out-patient, first rose to $48.9 \%$ in 2003 , and gradually declined to $27.3 \%$ in 2013 since 2008 . In terms of non-inpatient, the overall rate of non-inpatient who should be hospitalized is decreasing, from $35.9 \%$ in 1993 to $17.1 \%$ in 2013 , of which the decline in rural areas is much higher than that in urban areas. In terms of inpatients' behavior, the factors of self-recognition of minor illness have always played the most important constraint under the traditional medical concept of "taboo disease and taboo medical treatment". In the aspect of the disregard of the law of disease occurrence, the health and ill health of rural individuals have negatively reduced the outpatient rate, but the unhealthy factors have no significant impact, that is, cultural concepts have affected the outpatient rate of residents. However, Whether healthy or not, urban residents showed significantly lower outpatient tendency. In a word, from the perspective of non-outpatient who should be outpatient and non-inpatient who should be hospitalized, cultural tradition environments have a greater impact on medical values of urban residents than that of rural residents. Affected by traditional culture environment, urban residents used to disregard the occurrence of diseases before. With the increase of basic medical insurance and income, they now attach importance to prevention, but have not formed good medical values, and the phenomenon of "taboo disease and avoid medical treatment" is still serious. However, the medical value of rural residents has not changed much.

In terms of disregard for the laws of life course, from 6 follow-up surveys from 2000 to 2014, the proportion of samples of deaths at home decreased from $90.10 \%$ to $81.08 \%$, while the proportion of samples of deaths in medical institutions rose from $5.66 \%$ to $12.16 \%$, showing an obvious upward trend, that is, the number of the elderly dying in hospitals is finally increasing. The number of the days used for attending the elderly in the last month before the death increased from an average of 13.3109 days in 2000 to 23.2733 days in 2011. The total medical expenses of the elderly in the month before death decreased significantly in 2002, and began to rise again in 2005, that is, the basic medical insurance system and income growth effects have initially appeared. Income and policy interventions have an impact on the older people's end-of-life medical consumption, of which the empirical tests show that income levels have significantly increased the level of medical expenditures in the month 
before the end of life, and significantly increased the probability of deaths of the elderly in medical institutions. After the treatment of instrumental variables, the test results show that income still has a significant positive effect on medical consumption of the elderly before the death, and the coefficient value is higher, but there is no significant effect on the place chosen for death of the elderly. After controlling the undertaker of the medical consumption before the death of the elderly, we examine what impact the policy interventions have on the hospice medical consumption and the location of the dying. The test results show that the medical treatment expenses of end-of-life medical care is significantly lower for the elderly whose health care expenses are borne by their spouse or children living together. When the state or collective provides subsidy for medical expenses, the elderly who have no money to pay for medical consumption will also have significantly lower end-of-life medical expenditures, that is, income is still the core factor determining the medical consumption of this group, and policy interventions have not effectively improved their medical behavior or medical consumption values. In addition, when the medical costs are borne by the cooperative medical system, the probability of the elderly dying in hospital at the end of their life is significantly reduced. When the medical expenses are covered by the National Medical Insurance Fund, medical insurance, state or collective subsidized medical expenses, and at the same time the elderly themselves have no money to pay for medical expenses, the probability of dying in a medical institution is significantly higher for the elderly.

Based on the above conclusions, the article explores how to construct effective health management policy and medical consumption guidance from two aspects of health value concepts and medical value values, so as to promote citizens to form better medical consumption habits and health management concepts. Among them, health management policies should focus on the protection of healthy and unhealthy people in the whole social group, and the core is the implementation and guarantee of health management policies; while the medical value should play a protective role for unhealthy groups, the core of which is how to achieve Intervention protection of basic medical insurance policies and income policies. 


\section{Availability of data and materials}

The original data of the article comes from:

https://opendata.pku.edu.cn/dataset.xhtml?persistentId=doi:10.18170/DVN/XRV2WN

\section{Funding}

Funding:National Natural Science Fund of China (71904167) and Zhejiang Philosophy and Social Science Planning Project (20NDQN302YB)

\section{Authors' contributions}

Huan Liu revised it critically for important intellectual content and approved the version to be published,and carry out language retouching, modification.Tiantian $\mathrm{Hu}$ made a substantial contribution to the concept and design of the work, interpretation of data,and drafted the article.

\section{Acknowledgements}

The authors are very grateful for the financial support of National Natural Science Fund of China ( 71904167 ) and Zhejiang Philosophy and Social Science Planning Project (20NDQN302YB).

\section{References}

[1] Porter,M. E.;Teisberg,E. O.Redefining Health Care: Creating Value-based Competition on Results.Boston: Harvard Business Press.2006.

[2] Porter, M. E.What Is Value in Health Care?.New Engl J Med.2010, 363(26),2477-2481.

[3] Wang,D.Reform and innovation of medical insurance payment system based on value medical treatment.Chinese Soc Secy Rev.2019,3 (3) ,92-103.

[4] Pan, D. Medical Concepts, Social Cognition and Cultural Beliefs in Drug Gifts.J Guangxi Univ for Nationalities(Phi Soc Sci Edit).2017,39(1),11-19. 
[5] Long ,W. The medical elite of the Republic of China "Reinventing the patient".Soc Sci Front.2013, (6) ,84-91.

[6] Harold ,B.China and Modern Medicine: A Study in Medical Missionary Development.London: United Council for Missionary Education.1921.

[7] Delbanco ,T. L.Enriching the Doctor-patient Relationship by Inviting the Patients Perspective.Ann Intern Med.1992,116 (5) ,414-418.

[8] Pan ,DW. Patients, Families and Doctors in "Twenty-Four Filial Pieties"-A Medical Sociological Survey of Disease-Related Behaviors.Open Times.2015, (1) 109-117.

[9] Porter, R.Patients and Practitioners : Lay Perceptions of Medicine in Pre-Industrial Society.Cambridge University Press.2003.

[10] Segall, A.The Sick Role Concept: Understanding Illness Behavior.J Health Soc Behav.1976,17(2),162-169.

[11] Liu, Z.Y. "Two Solutions to Divine Medicine": Baima Tibetan's Folk Medical Concept and Practice.J Southwest Minzu Univ(Hum Soc Sci).2009,29（10）,14-21.

[12] World Health Organization. Primary Health Care.Geneva:World Health Organization.1978.

[13] Baer ,H. A . PERSPECTIVES: On the Political Economy of Health.Med Anthropol Q.1982,14(1),1-17.

[14] Lin ,K. M . Social Origins of Distress and Disease: Depression, Neurasthenia, and Pain in Modern China. Psychosomatics.1987,28(8),445-446.

[15] Woolhandler ,S. ;Himmelstein,D,U. Ideology in medical science: Class in the clinic.Soc Sci Med .1989,28(11),1205-1209.

[16] Singer, M . Anthropology and addiction: an historical review. Addiction.2012,107(10),1747-1755.

[17] Frankenberg, R.Global Health Policy, Local Realities: The Fallacy of the Level Playing Field.Med Anthropol Q.2001,15(2),269-273.

[18] Liu ,F.S.We need a revolution in medical concepts-thinking about cultural medicine and species medicine.Med Philos.2008,29 (6) ,7-11.

[19] Häkkinen, U ; Rosenqvist, G ;Aro, S. Economic depression and the use of physician services in Finland. Health Econ.1996,5(5),421-434. 
[20] Cameron ,A .C; Trivedi, P. K ; Piggott, F. M . A Microeconometric Model of the Demand for Health Care and Health Insurance in Australia. Rev Econ Stud.1988,55(1),85-106.

[21] Gerdtham ,U. G . Equity in Health Care Utilization: Further Tests Based on Hurdle Models and Swedish Micro Data. Health Econ.1997, 6(3),303-319.

[22] Deb ,P ; Trivedi, P. K . The structure of demand for health care: latent class versus two-part models. J Health Econ.2002,21(4),601-625.

[23] Akin, J. S; Griffin, C. C.; Popkin, G. B. M . The Demand for Primary Health Care Services in the Bicol Region of the Philippines. Econ Dev Cult Change.1986,34(4),755-782.

[24] Gertler, P. J ; Locay, L.; Sanderson, W. C . Are User Fees Regressive ? The Welfare Implications of Health Care Financing Proposals in Peru.J Econom.1987,36(Supp),67-88.

[25] Ching ,P . User fees, demand for children's health care and access across income groups: The Philippine case. Soc Sci Med.1995,41(1),37-46.

[26] Gupta, S. ; Verhoeven, M.; Tiongson, E. R .The effectiveness of government spending on education and health care in developing and transition economies. Eur J Polit Econ. 2002,18(4),717-737.

[27] Ulrich ,P .V . An Econometric Model of the Two-Part Decision making Process in the Demand for Health Care. J Hum Resour.1995,30(2),339-361.

[28] World Bank."Reviewing the Challenges Facing China's Rural Health Work", World Bank "China Rural Health Briefing" series.2005.

[29] Van Doorslaer, E. ; Wagstaff ,A.; Bleichrodt, H.;et al. Income-related inequalities in health: some international comparisons.J Health Econ.1997,16(1),93-112.

[30] Judge ,K. ; Mulligan, J. A. ; Benzeval, M. The relationship between income inequality and population health. Soc Sci Med.1998,47(7),983-985.

[31] Wagstaff ,A. ; Lindelow, M . Can insurance increase financial risk?: The curious case of health insurance in China. J Health Econ.2008, 27(4),1-16.

[32] Wagstaff, A.Poverty and Health Sector Inequalities.Bull World Health Organ.2002,(2),97-105.

[33] Dollar, D;Kraay, A.Growth is Good for the Poor.J Econ Growth.2002, (3) ,195-225. 
[34] Feng, J.;Yu, Y.Y. Income disparity and health in rural China.Econ Res J.2007, (1 ),79-88.

[35] Xie ,E. Medical Insurance and Anti-Poverty Urban and Rural: 1989-2006.J Financ Econ.2008,34 (12),68-83.

[36] Alleyne ,G. A. O. ; Casas, J. A. ; Castillo-Salgado, C . Equality, Equity: Why Bother?. Bull World Health Organ.2000,44（4）,76.

[37] Evans, T. M;Whitehead, F.;Diderichsen, A.Challenging Inequities in Health:From Ethics to Action.Oxford: Oxford University Press.2001.

[38] Xie, E.Research on income-related health and medical service use inequality.Econ Res J.2009,（2）,92-105.

[39] Blakely, T. A . What is the lag time between income inequality and health status?. J Epidemiol Commun H.2000,54(4),318-319.

[40] Subramanian, S. V.; Ichiro, K.Income Inequality and Health:What Have We Learned So Far?. Epidemiol Rev .2004,26,78-91.

[41] Mellor, J. M; Milyo, J . Income Inequality and Health Status in the United States: Evidence from the Current Population Survey. J Hum Resour.2002, 37(3),510-539.

[42] Krugman, P.The Spiral of Inequality, Mother Jones(November December).1996,44-49.

[43] Deaton, A.Health, Inequality,and Economic Development. J Econ Lit.2003,41(1),113-158.

[44] Wilkinson, R. G.Unhealthy Societies: The Afflictions of Inequality. Biochem.1998,10(8),1335-1339.

[45] Kawachi, I. ; Kennedy, B. P . Income Inequality and Health: Pathways and Mechanisms. Health Serv Res.1999,34(1 Pt 2),215-227.

[46] Grand, J. L . Equity,health,and health care. Soc Justice Res.1987,1(3),257-274.

[47] Treasury, T. Poverty, Income Inequality and Health. Treasury Working Paper.2001.

[48] Braveman, P.Challenging Inequities in Health: From Ethics to Action. Bull World Health Organ.2002,80(2),1-15.

[49] Tang, J.; Li ,J.Holistic Health Concept and Health Management from the Perspective of Health Sociology.Soc Sci in China.2019,(8),130-148.

[50] Tang,J.Lifestyle and overall health concept [J / OL]. J Harbin Institute Techno (Soc Sci Edit).2020,(01),38-44 [2020-03-03].https://doi.org/10.16822/j.cnki.hitskb.2020.01.006. 
[51] Jin ,C.L.;Wang, H.Y.;Sun, H.;et al. The concept, practice and realization path of value medicine.Health Econ Res.2019,36(02),6-8.

[52] Ma,C.;Yu ,Q.W.;Song, Z.; et al.Long-term Nursing Insurance, Medical Expense Control and Value Medical.China Indus Econ.2019,(12),42-59.

[53] Gu, X.F.; Liu ,X.Q.From quantity to value: how medical insurance payment drives the integration of medical and health systems.Health Econ Res.2020,37(01),7-10. 